\title{
Simplified Parquet Equations for the Anderson Impurity Model: Comparison with Numerically Exact Solutions
}

\author{
V. POKORNÝ ${ }^{a}, *$, M. ŽONDA ${ }^{b}$, A. KAUCH ${ }^{a}$ AND V. JANiŠ $\check{K}^{a}$ \\ ${ }^{a}$ Institute of Physics, Czech Academy of Sciences, Na Slovance 2, 18221 Prague, Czech Republic \\ ${ }^{b}$ Department of Condensed Matter Physics, Faculty of Mathematics and Physics, Charles University in Prague, \\ Ke Karlovu 5, 12116 Prague, Czech Republic
}

\begin{abstract}
We use an analytic solver for the single-impurity Anderson model based on simplified parquet equations to describe the Kondo asymptotics. This scheme uses a two-particle self-consistency to control the strong-coupling Kondo critical behavior of this model at half filling. The equations can be written in the real-frequency representation, which gives us direct access to spectral functions unlike numerical schemes in the Matsubara formalism. We compare our results to those obtained by second-order perturbation theory, numerical renormalization group, and continuous-time quantum Monte Carlo in order to assess the reliability of this approximation.
\end{abstract}

DOI: 10.12693/APhysPolA.131.1042

PACS/topics: 71.10.Fd, 72.15.Qm, 74.40.Kb

\section{Introduction}

The properties of weakly and intermediately correlated electrons are captured sufficiently well by the (perturbative) Fermi liquid theory. However, with increasing interaction strength the system can become unstable due to quantum fluctuations. One then needs to employ nonperturbative approaches to describe the properties close to the emerging quantum critical point of the system of strongly correlated electrons.

One option is to use numerical techniques such as exact diagonalization, quantum Monte-Carlo or the numerical renormalization group (NRG). These methods are usually both time and computational resources consuming and each of them has its specific limitations. Other option is to use analytic methods, usually based on the perturbation expansion in the interaction strength. Unfortunately, one needs to sum up an infinite series of diagrams in order to access the strong-coupling regime. Furthermore, since the quantum criticality is marked by divergences in the two-particle and response functions, such as susceptibilities, the calculation scheme must incorporate two-particle self-consistency to maintain control over the divergent behavior.

One example of an analytic method was recently described in Ref. [1] and represents an extension of the scheme developed earlier in Refs. [2, 3]. It employs simplified parquet equations (SPE) method with only static renormalization of one-particle propagators to determine self-consistently the two-particle vertices. In order to maintain the consistency between the divergent behavior of the two-particle vertices and the symmetry breaking in one-particle functions, this scheme defines two self-energies. The so-called thermodynamic self-energy is

*corresponding author; e-mail: pokornyv@fzu.cz used to renormalize propagators in the two-particle equations and serves only as an auxiliary function breaking the symmetry at the critical point. The spectral selfenergy on the other hand determines the shape of the spectral function and the physical properties of the system.

The purpose of this contribution is to assess the reliability of this approximation by comparing the spectral properties of a simple model with results obtained by a set of other established, both numerical and analytic methods.

\section{The model and the method}

We test the construction of the solver on the one-band single-impurity Anderson model (SIAM) which describes a magnetic impurity embedded in a metallic host. The quantum critical point lies at infinite interaction strength and is generated by magnetic spin-flip fluctuations leading eventually to the breaking of the spin symmetry [4]. The model Hamiltonian is

$$
\begin{aligned}
\mathcal{H} & =\sum_{k \sigma} \varepsilon_{k} c_{k \sigma}^{\dagger} c_{k \sigma}+U d_{\uparrow}^{\dagger} d_{\uparrow} d_{\downarrow}^{\dagger} d_{\downarrow} \\
& -\sum_{\sigma} \mu_{\sigma} d_{\sigma}^{\dagger} d_{\sigma}+\sum_{k \sigma}\left(V c_{k \sigma}^{\dagger} d_{\sigma}+V^{*} d_{\sigma}^{\dagger} c_{k \sigma}\right),
\end{aligned}
$$

where $c_{k \sigma}^{\dagger}$ creates a conduction electron with dispersion $\varepsilon_{k}$ and spin $\sigma=\uparrow$ or $\downarrow, d_{\sigma}^{\dagger}$ creates an electron with spin $\sigma$ at the impurity site with energy $E_{d}, \mu_{\sigma}=-E_{d}+\sigma h$ is the spin-dependent chemical potential containing the external magnetic field $h, U$ is the local Coulomb interaction and $V$ is the hybridization strength between the conduction band and the impurity site. Assuming constant density of states $\rho_{0}=(2 D)^{-1}$ of the conduction band with a half-bandwidth $D$, the non-interacting local impurity Green function takes a Lorentzian form $G_{\sigma}^{(0)}(\omega)=\left(\omega+\mu_{\sigma}+\mathrm{i} \Delta\right)^{-1}$ with the width $\Delta=\pi \rho_{0} V^{2}$, where $\omega$ is the (real) frequency. 
We calculated the spectral functions of SIAM in a wide region of interaction strengths using our implementation of SPE. The approach described in Ref. [1] reduces a set of coupled integral equations to a self-consistent set of algebraic ones for the regular part $\Lambda$ and the singular part $K$ of the two-particle vertex. At zero temperature and zero magnetic field they read

$$
\Lambda=\frac{U}{1+\psi}, \quad K(\omega)=-\frac{\Lambda^{2} \phi(\omega)}{1+\Lambda \phi(\omega)},
$$

where

$$
\begin{aligned}
& \psi=-\int_{-\infty}^{0} \frac{\mathrm{d} x}{\pi} \operatorname{Im}\left(G(x) G(-x) K^{*}(-x)\right), \\
& \phi(\omega)=-\int_{-\infty}^{0} \frac{\mathrm{d} x}{\pi}\left(G(x+\omega)+G^{*}(x-\omega)\right) \operatorname{Im} G(x),(3)
\end{aligned}
$$

and $G(\omega)$ is the impurity Green function renormalized by the thermodynamic self-energy $\Sigma^{T}$. We dropped the spin label $\sigma$ since we are interested in the spin-symmetric solution only. The frequency variable $\omega$ is assumed to have an infinitesimal positive imaginary part $\omega \rightarrow \omega+\mathrm{i} 0^{+}$. The thermodynamic self-energy is calculated from the vertex via a Ward identity linearized with respect to the external magnetic field $h$, which is then set to be zero. This identity reflects the conservation of charge. The spectral self-energy $\Sigma^{S}$ is determined from the dynamical, Schwinger-Dyson equation. The two self-energies are

$$
\begin{aligned}
& \Sigma^{T}=-\Lambda \int_{-\infty}^{0} \frac{\mathrm{d} x}{\pi} \operatorname{Im} G(x), \\
& \Sigma^{S}(\omega)=-U \int_{-\infty}^{0} \frac{\mathrm{d} x}{\pi} \frac{\operatorname{Im} G(x)}{1+\Lambda \phi(x-\omega)} \\
& \quad-\Lambda G(x+\omega) \operatorname{Im} \frac{\phi(x)}{1+\Lambda \phi(x)} .
\end{aligned}
$$

The interacting Green function $G^{S}(\omega)$ is then obtained from the spectral self-energy using the Dyson equation. All these equations are in a form of frequency convolutions and can be calculated very efficiently using the Fourier transform, so the converged solution can be obtained within seconds on a standard PC.

To compare the results of SPE with other methods, we also calculated the spectral functions using NRG, quantum Monte Carlo and second-order perturbation theory (PT). NRG data at zero temperature were obtained using the NRG LJUBLJANA code [5]. We used the "selfenergy trick" to reduce NRG over-broadening effect [6]. The continuous-time hybridization-expansion (CT-HYB) quantum Monte Carlo calculations were performed using the solver included in TRIQS package [7]. Since this method works only at finite temperatures, we set $k_{\mathrm{B}} T=0.0125 \Delta$. The CT-HYB spectral functions were obtained by analytic continuation of the imaginary-time Green function using the maximum entropy method [8]. Finally, we calculated the spectral functions using the simple second-order PT, to see the breakdown of the finite-series summation technique for strong interactions.

\section{Results}

From now on we resort to the particle-hole symmetric case, where the thermodynamic self-energy is compensated by the chemical potential $\mu=-E_{d}+U / 2$ and we use $\Delta$ as the energy unit in all calculations.

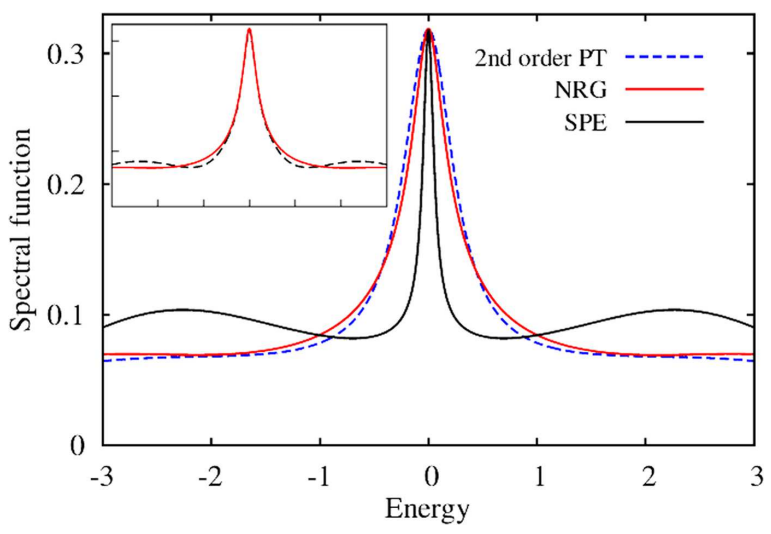

Fig. 1. Spectral function of the one-band Anderson impurity model at zero temperature for interaction strength $U=6 \Delta$ at half-filling calculated by different methods: simplified parquet equations (black), secondorder PT (blue dashed) and NRG (red). Inset shows the comparison between NRG (red) at $T=0$ and CTHYB quantum Monte Carlo at $k_{\mathrm{B}} T=0.0125 \Delta$ (black dashed).

In Fig. 1 we plotted the spectral function $\rho(\omega)=$ - Im $G^{S}(\omega)$ for $U=6 \Delta$ calculated by various methods. The inset shows the comparison between NRG and CTHYB. The CT-HYB result shows a bit more developed Hubbard satellites, but the important shape of the Kondo resonance peak at the Fermi energy is the same in both methods. The slight reduction of the height of the central peak in the CT-HYB solution is due to the finite temperature effects. The spectral functions obtained with these two numerical methods are in good agreement, we henceforth use the NRG as the benchmark, since it is suitable for the zero-temperature case.

Since the interaction is not very strong, the simple second-order PT approximates the NRG result well. This ability of the non-self-consistent second-order PT to provide quantitatively reliable results in a wide range of parameters is discussed in detail in Ref. [9].

The spectral function obtained by SPE shows the canonical three-peak structure with a Kondo resonance at the Fermi energy, although the width of the central peak is significantly smaller and the Hubbard satellites are more developed than in the NRG spectral function.

The critical region of SIAM is characterized by asymptotic vanishing of the Kondo scale. In Fig. 2 we plotted the Kondo scale defined as the half-width at halfmaximum of the Kondo resonance peak obtained by different schemes. Both NRG and SPE results show exponential behavior of the Kondo scale for large interaction strengths $U / \Delta$. Furthermore, both these methods give 


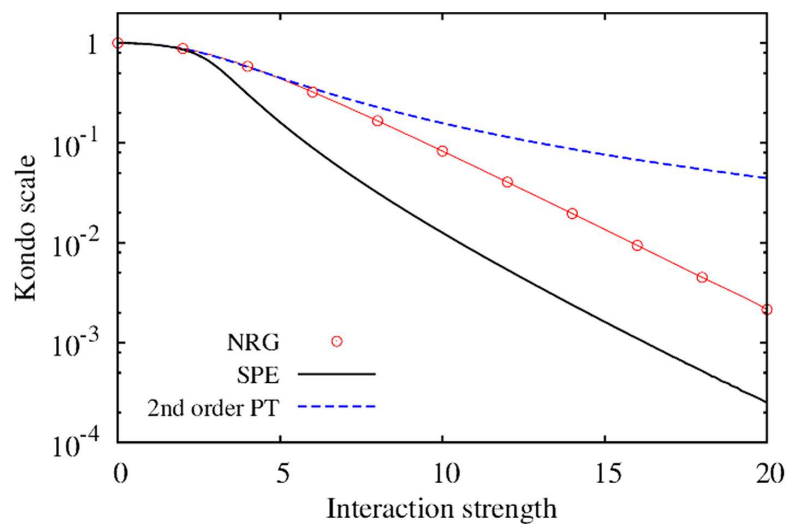

Fig. 2. Comparison of the Kondo scale at zero temperature defined as the half-width at half-maximum of the central peak in spectral function as a function of interaction strength $U / \Delta$. Blue dashed: second-order PT, black: SPE, red: NRG. Note the logarithmic scale on the vertical axis.

correct scaling factor within the numerical accuracy when compared to the exact, Bethe-ansatz solution which gives the universal scaling factor $\exp (-\pi U /(8 \Delta))$.

On the other hand, SPE underestimates the width of the resonance, as already shown in Fig. 1, while NRG gives the correct width, which we tested by comparison with CT-HYB and the exact solution. The source of this discrepancy is not yet known and will be a subject of future research.

The second-order PT, despite giving reasonable quantitative results up to $U / \Delta \approx 10$, fails to provide the correct scaling, and is not able to describe the Kondo behavior. This shows the well-known fact that one needs to sum up an infinite subset of terms in the perturbation expansion series to obtain correct behavior close to the quantum critical point.

\section{Conclusions}

We presented a comparison of the method based on simplified parquet equations developed in Ref. [1] with other well-known methods to assess its reliability in the description of quantum criticality in systems of strongly correlated electrons. We used the simple oneband single-impurity Anderson model as an example and presented spectral functions and the behavior of the Kondo scale in the strong-coupling limit. The impurity solver based on the simplified parquet equations provides us with a qualitatively correct structure of the spectral function, interpolates well between weak and strongcoupling regimes and it is free of any unphysical symmetry breaking leading to spurious magnetic solutions. Furthermore, we obtain a correct exponential scaling of the width of the quasiparticle peak with increasing interaction strength. Despite the fact that this method fails to produce quantitatively correct values of the Kondo scale, it represents a consistent and affordable description of quantum criticality in correlated electron systems.

\section{Acknowledgments}

Research on this problem was supported by grant No. 15-14259S of the Czech Science Foundation.

\section{References}

[1] V. Janiš, A. Kauch, V. Pokorný, Phys. Rev. B 95, 045108 (2017).

[2] V. Janiš, P. Augustinský, Phys. Rev. B 75, 165108 (2007).

[3] V. Janiš, P. Augustinský, Phys. Rev. B 77, 085106 (2008).

[4] A.C. Hewson, The Kondo Problem to Heavy Fermions, Cambridge University Press, Cambridge 1993.

[5] R. Žitko, NRG LJUBLJANA, open source numerical renormalization group code.

[6] R. Bulla, A.C. Hewson, T. Pruschke, J. Phys. Condens. Matter 10, 8365 (1998).

[7] P. Seth, I. Krivenko, M. Ferrero, O. Parcollet, Comp. Phys. Commun. 200, 274 (2016).

[8] M. Jarrell, J. Gubernatis, Phys. Rep. 269, 133 (1996).

[9] J. Gukelberger, L. Huang, P. Werner, Phys. Rev. B 91, 235114 (2015). 